\title{
Monte Carlo Method and Brownian Movement Applied to Future Stock Market Analysis
}

\author{
Fabio Lopes Licht \\ UCP/UNESA \\ Petropolis - RJ \\ Brazil
}

\begin{abstract}
This paper presents a method of forecasting the return on investment on the stock exchange based on the historical analysis of the stock and the generation of massive random data. The model is based on the use of the Monte Carlo method and on the Simulation of the Brownian Movement with a massive generation of data to generate forecasts of growth or decrease in the value of the stock over a given time. As a model test, stock market indices in Brazil and historical stock data were used.
\end{abstract}

\section{General Terms}

Monte Carlo Method, Brownian Movement

\section{Keywords}

Monte Carlo Method, Market Activity, Brownian Motion

\section{INTRODUCTION}

The economic stability followed in recent years in Brazil and in the world has resulted in an increase in the number of investments in the stocks exchange at the expense of other more stable applications with less profit. What was almost an exclusivity of the upper class, is now common to many types of investors who seek in the stock market a chance to multiply their financial resources in less time than those made in investments such as savings, which is more popular in Brazil.

It even with this increase in the number of investors and economic stability, the risk has not diminished and for the small investor, they either opt for an investment company that charges their fees, or studies the stock market, an option with a very long learning curve, or searching tools that predict profit, or loss, over the expected investment time. This paper aims to collaborate with the small investor in that it predicts the percentage of profit or loss estimated over a period in an investment whose decision making is totally uncertain.

To achieve this proposal, this article presents the concepts of the Monte Carlo Method, the Simulation of the Brownian Movement, and a brief description of the functioning of IBOVESPA.

\section{LITERATURE REVIEW}

In this section, the technologies involved in the prototype and related works are presented.

\subsection{Monte Carlo Method}

The Monte Carlo Method consists of generating many numbers to simulate future projections [3]. Through this method, it is possible to generate several numbers and obtain statistics from those we interest in the problem. The most common example of using this method is to calculate the value of the area by estimate, where points are generated within a square and the probability of these points being within the polynomial area is calculated, that is, how many points were drawn in the area of the total points generated. Thus, the greater the number of points, the closer to the actual area value.

\subsection{Simulation of the Brownian Movement}

The Simulation of the Brownian Movement is a stochastic process based on randomness, it was initially observed by Robert Brown in 1826 who thought that the movement of the pollen observed by the microscope was caused by this living being, only afterward it was verified that the movement was due to the collision pollen with air molecules [4] [12] [10]. One of the purposes of this method is precisely the analysis of changes in assets in the market, which allowed us to simulate the future analysis of shares on the stock exchange, using the Monte Carlo method.

2.2.1 Excerpt from source code of the Brownian Movement. The script that simulates the Brownian motion is shown in the code snippet, where " $\mathrm{n}$ " is a very large value. This number " $\mathrm{n}$ " represents the massive amount of random values generated.

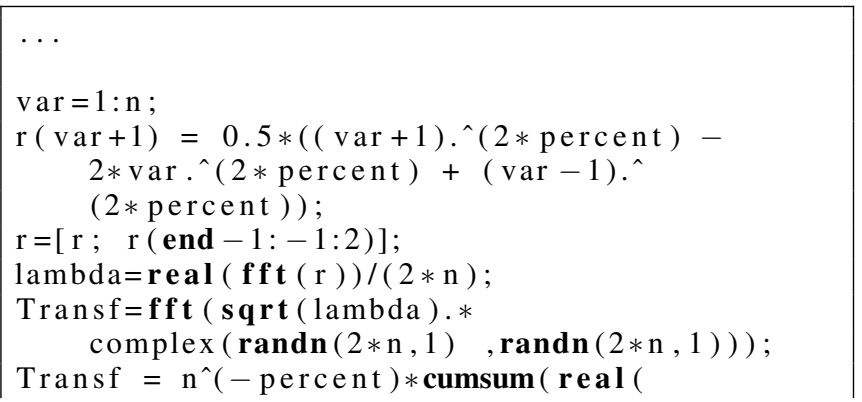


Transf $(1: \mathrm{n}+1)))$

Transf $=100^{\wedge}$ percent $*$ Transf ;

$\cdots$

\subsection{Ibovespa}

According to B3 (Brazil, Stock Exchange, Tour), in Portuguese (Brasil, Bolsa, Balcao), [7]: Ibovespa is the main performance indicator of the shares traded at B3 and brings together the most important companies in the Brazilian capital market. It was created in 1968 and, over those 50 years, it has consolidated itself as a reference for investors around the world. Reassessed every four months, the index is the result of a theoretical portfolio of assets. It consists of the shares and units of companies listed on B3 that meet the criteria described in its methodology, corresponding to approximately $80 \%$ of the number of deals and the financial volume of our capital market.

\subsection{Related Work}

The use of both methods Monte Carlo and Brownian Movement is noted in the article [6]. In this article, the authors analyze the methods in a basic way applied to stock prices. The authors present a Markov process and identify that the stock price follows a random walk, and the recent price information is already incorporated so that the next price movement is "conditionally independent". This article served as a basis for the generation of a model that would allow us to create an analysis for the Brazilian stock market, the Ibovespa in a more complete way.

\section{DEVELOPMENT}

It has defined the path for the creation of an algorithm for forecasting future values, we opted for the use of the MatLab language as it contains several pre-programmed functions that facilitate the prototype construction, such as massive random generation and graph construction.

\subsection{Excerpts from the simulation source code}

The following code snippets present the generation of massively random values, as proposed by the Monte Carlo Method, with the use of the Brownian Movement still within the limit of the proposed values, that is, the lowest and highest historical percentages acquired from the values shares on the stock exchange. These random values form a knowledge base to propose a scale of acceptable values for the problem, however, the algorithm is always based on the value immediately preceding, since the next step is random and generated only and exclusively from the last value of the stock's action. analyzed stock exchange.

In the first block of the code snippet, an estimated value is generated based on the Brownian Movement, normalized using the MaxMin normalization equation presented in the Ph.D. thesis [8]. In the second code snippet, the Dickey-Fuller test increased in the time series is applied to reject the null hypothesis that there is a unit root in the series. In the third code section, the model is validated with the adjustment of the polynomial curve, seeking to return the coefficients for a polynomial $\mathrm{p}(\mathrm{x})$ of degree $\mathrm{n}$, considered the best fit for the data.
Volume 175 - No.34, December 2020

$\mathrm{a}=$ BrownianMoviment $(0.3,2 \wedge 15) * 100$;

for $\mathrm{i}=1$ : days estimate $($ i $)=$ $($ a $($ i $)-\min ($ a $)) /(\max (a)-\min ($ a $))$ $*($ difference $*$ Index Analyzed $($ qtd -1$))+$

end Index Analyzed ( qtd)* LowestIndex ;

for $i=q t d+1: q t d+d a y s$

IndexAnalyzed (i $)=$ estimate $(i-q t d)$;

end

$\cdots$

ste $\mathrm{p}=6$

TempIndex $=$ detrend (Index Analyzed );

TrendIndex =Index Analyzed -TempIndex;

$\mathrm{H} 1=\mathrm{adfte} \mathrm{s}(\mathrm{TempIndex})$;

$\cdots$

TempIndex $=[$ TempIndex $; \operatorname{zeros}($ ste $p, 1)]$; $\mathrm{n}=\mathrm{idd}$ at a (TempIndex $)$;

$\mathrm{m}=\operatorname{armax}($ IndexObject,, IndexTestI

Index TestII ]);

$\mathrm{P} 1=$ predict $(\mathrm{m}, \mathrm{n}, 1)$;

Predict $=$ P1. OutputData ;

Predict $=$ Predict';

if size (DerivativeIndex, 2$)^{\sim}=0$

for Index $=\mathbf{s i z e}($ DerivativeInde $x, 2):-1:$ Predict $=$ cumsum $([$ DerivativeIndex (Index ), Predict ]); end

end

m=polyfit ([ $1:$ size (IndexTrend , 2)], IndexTrend, , 1);

$\mathrm{xt}=[]$;

for $\mathrm{j}=1$ : step $x t=[x t$, size $($ IndexTrend, 2$)+j]$;

end

TrendResult $=$ polyval $(\mathrm{mp} 1, \mathrm{xt})$;

PreData $=$ TrendResult + Predict (size (

IndexAnalyzed , 2) +1 : size (Predict ,2));

$\mathrm{x}=[$ IndexTrend ', TrendResult $]+$ Predict ;

rate $=x($ length $(x)) / x($ qtd $)$;

average $($ acao $)=\operatorname{average}(\operatorname{acao})+(($ rate -1$) * 100)$

$\cdots$

It is the first step in the preparation of the source code in MatLab was to obtain the history of stock quotes on the Ibovespa website, for this, a ready-made spreadsheet was available on the Investor Tools [5] website. This implementation allows, through the "Ticker" to download all stock updates between two dates. There are several websites that make this data available for different stock exchanges, which allows the model to be used for other stock exchanges in other countries. The actions chosen to test the implementation were those shown in Table 3.1

After the acquisition of historical data of IBOVESPA obtained from the website, it was decided to define lower and upper date limits, from 06/18/2012 to the present date, considering that YDUQS 
Table 1. Ibovespa Stock Table

\begin{tabular}{|l|l|l|}
\hline Ticker & T. Yahoo & Name \\
\hline$I B O V$ & ${ }^{\text {BVSP }}$ & Ibovespa \\
\hline USDBRL & USDBRL=X & Dollar \\
\hline ABEV3 & ABEV3.SA & Ambev \\
\hline GGBR4 & GGBR4.SA & Gerdal \\
\hline VALE3 & VALE3.SA & Vale \\
\hline PETR3 & PETR3.SA & Petroleo Brasileiro SA Petrobras \\
\hline YDUQ3 & YDUQ3.SA & YDUQS \\
\hline
\end{tabular}

shares started to be offered on stock exchange quotations on this date.

It analyzes each of the actions presented in Table 3.1, the highest daily index and the lowest daily index were found throughout the history, where the highest represents gain, as it is above 1 and the lowest represents loss, where the index is below 1 As an example, one can observe the Ibovespa Index, which had its highest historical increase (13.91\%) in the period mentioned (06/18/2012) until $07 / 08 / 2020$ (the last test carried out with the model), and its highest loss of value $(-14.78 \%)$ in the same historical series analyzed.

It is based on these minimum and maximum values obtained, it was possible to predict, normally, there would be no variation greater or less than these and it would be possible to make an analysis based on the last quote, raising or lowering the value of the shares within these limits, based solely and exclusively on the last stock value, since a random model is independent of previous values.

To force the model and create a stock performance analysis, a temporary index was used, removing the nth degree polynomial trend. The trend of the index, in this case, will be the index analyzed minus the temporary index. The temporary index will still be used to study the regression of the time series.

The Dickey-Fuller test [11], which allows the creation of non-null hypotheses by returning a logical value with the rejection decision in a univariate time series. If the test returns a null hypothesis, the derivative is applied to the temporary index and again the DickeyFuller test is used. It is worth mentioning that during the tests, only the dollar price index needed to be derived and this was because there was a constant increase in value and rare decreases as occurs naturally with stocks. The analysis with shares and with the dollar will be presented in the results, where it will be explained why the model created does not serve to analyze currencies.

For the prediction and comparison of the model with the real data, ARIMA was used [1]. ARIMA is an algorithm that allows us to adjust integrated autoregressive models of moving averages [9].

There are several prediction models in the literature using the ARIMA algorithm, but the vast majority have used the algorithm together with artificial neural networks for forecasting. No model was found by the author to make a prediction based on the generation of random numbers using the ARIMA algorithm.

Still, on random numbers, those generated for the model do not simply follow the generation of pseudo-random numbers, but numbers were generated using fractional Brownian motion (FBM) using the Fourier fast transform [2]. FBM allows modeling of stochastic processes with great statistical similarity and for this reason, it was chosen for use in the prediction model of this work.

\begin{tabular}{|c|c|c|c|c|c|c|}
\hline \multirow{2}{*}{ Ticker } & \multicolumn{3}{|c|}{ Number of days } & \multicolumn{3}{|c|}{ Forecast accuracy } \\
\hline & 100 & 500 & 1000 & 100 & 500 & 1000 \\
\hline$I B O V$ & $-2,71 \%$ & $14,43 \%$ & $13,13 \%$ & $88,89 \%$ & $89,76 \%$ & $89,39 \%$ \\
\hline USDBRL & $2,32 \%$ & $4,88 \%$ & $14,34 \%$ & $0,89 \%$ & $91,44 \%$ & $90,85 \%$ \\
\hline$A B E V 3$ & $27,35 \%$ & $5,72 \%$ & $10,88 \%$ & $88,12 \%$ & $90,73 \%$ & $89,54 \%$ \\
\hline GGBR4 & $-14,67 \%$ & $4,29 \%$ & $7,09 \%$ & $91,49 \%$ & $92,03 \%$ & $91,84 \%$ \\
\hline VALE3 & $-0,78 \%$ & $6,54 \%$ & $20,06 \%$ & $0,31 \%$ & $91,83 \%$ & $92,55 \%$ \\
\hline PETR3 & $3,96 \%$ & $9,10 \%$ & $24,37 \%$ & $89,93 \%$ & $90,16 \%$ & $90,52 \%$ \\
\hline YDUQ3 & $3,04 \%$ & $5,38 \%$ & $22,82 \%$ & $88,44 \%$ & $88,49 \%$ & $89,50 \%$ \\
\hline
\end{tabular}

Table 3. Result of 30 Simulations

\begin{tabular}{|l|l|l|l|}
\hline \multirow{2}{*}{ Ticker } & \multicolumn{3}{|c|}{ Average of 30 simulations } \\
\cline { 2 - 4 } & $\mathbf{1 0 0}$ & $\mathbf{5 0 0}$ & $\mathbf{1 0 0 0}$ \\
\hline IBOV & $0,52 \%$ & $12,31 \%$ & $14,28 \%$ \\
\hline USDBRL & $-3,56 \%$ & $3,72 \%$ & $15,05 \%$ \\
\hline GGEV3 & $25,15 \%$ & $6,01 \%$ & $9,92 \%$ \\
\hline VALE3 & $-15,05 \%$ & $5,14 \%$ & $6,98 \%$ \\
\hline PETR3 & $-14,12 \%$ & $6,10 \%$ & $18,57 \%$ \\
\hline YDUQ3 & $4,25 \%$ & $9,33 \%$ & $23,48 \%$ \\
\hline
\end{tabular}

\section{RESULTS}

Based on what has been analyzed, this section seeks to present the result of three executions of the investment forecasting algorithm, the first with a 100-day forecast, the second with 500 days, and the third with 1000 days. The analysis is detailed in Table 3.1

To avoid biased results of the execution, 30 simulations of each action were performed and with the same number of days, the result that is shown in Table 4 was generated through the arithmetic mean of all the executions.

It can be seen between Table 3.1 and Table 4 that there were no major variations between the values generated in one run and the average of 30, and this can be understood as the demonstration that the model is adjusted and ready for more comprehensive tests, such as comparing the results generated with the actual results. Unfortunately, this approach requires time, considering that it will be necessary to wait 100,500 , and 1000 days to compare the results. If the possibilities of shorter times, 10, 20, and 30 days, for example, are considered, there will be a reduced wait, however, with a greater probability of errors, since there is the hypothesis of a phenomenon such as that of COVID-19 [13], responsible for great stock exchange variation, drop the stock values, for example. The 
Table 4. Expected value versus obtained value with a 100-day

\begin{tabular}{|c|c|c|}
\hline & \multicolumn{2}{|c|}{ Average of 30 simulations } \\
\hline \multirow[t]{2}{*}{ Ticker } & \multicolumn{2}{|l|}{100} \\
\hline & Expected value & Earned Value \\
\hline$I B O V$ & {$[-2,40 ;-3,01]$} & 0,52 \\
\hline USDBRL & {$[0,02 ; 4,61]$} & $-3,56$ \\
\hline$A B E V 3$ & {$[24,10 ; 30,59]$} & 25,15 \\
\hline GGBR4 & {$[-13,42 ;-15,91]$} & $-15,05$ \\
\hline VALE3 & {$[-0,00 ;-1,55]$} & $-14,12$ \\
\hline PETR3 & {$[3,56 ; 4,35]$} & 4,25 \\
\hline$Y D U Q 3$ & {$[2,68 ; 3,39]$} & 1,66 \\
\hline
\end{tabular}

Table 5. Expected value versus obtained value with a 500-day

\begin{tabular}{|l|l|l|}
\hline \multirow{2}{*}{ Ticker } & \multicolumn{2}{|l|}{ Average of 30 simulations } \\
\cline { 2 - 3 } & \multicolumn{2}{|l|}{500} \\
\cline { 2 - 3 } & Expected value & Earned Value \\
\hline \multirow{2}{*}{ IBOV } & {$[12,95 ; 15,90]$} & 12,31 \\
\hline USDBRL & {$[4,46 ; 5,29]$} & 3,72 \\
\hline ABEV3 & {$[5,18 ; 6,25]$} & 6,01 \\
\hline GGBR4 & {$[3,94 ; 4,63]$} & 5,14 \\
\hline VALE3 & {$[6,00 ; 7,07]$} & 6,1 \\
\hline PETR3 & {$[8,20 ; 9,99]$} & 9,33 \\
\hline YDUQ3 & {$[4,76 ; 5,99]$} & 5,62 \\
\hline
\end{tabular}

values of 100,500 , and 1000 were not chosen at random and the proposal was that it would be an analysis in approximately 3 months, 1 and a half years, and 3 years, considering that even if the daily values are different, during this period of time the tendency would be simulated, since non-standard values caused by disasters would be discarded, for example.

Tables 4, 4 and 4 presents a comparison between the values that were expected based on the prediction of correctness in Table 1 and the result of the same table with the average obtained from 30 simulations presented in Table 4 As a result, the values that were outside of what was highlighted were highlighted. was expected, most of them with a 100-day simulation, which shows that the model should be adjusted for short-term forecasts.

Still in the tables, it is possible, it can be seen that for a 500day simulation, only 2 values were outside of what was expected, the variation of the Dollar in relation to the Brazilian Real, whose model is not adequate, as it does not necessarily follow the movement of the stock market. of values, and the second, of the value of Gerdal's stock actions, in this case, no justification for the error was found, but it can be seen that for a 500-day forecast all values were
Table 6. Expected value versus obtained value werther 172820 a 1000 -day

\begin{tabular}{|l|l|l|}
\hline \multirow{2}{*}{ Ticker } & \multicolumn{2}{|l|}{ Average of 30 simulations } \\
\cline { 2 - 3 } & \multicolumn{2}{|l|}{1000} \\
\cline { 2 - 3 } & Expected value & Earned Value \\
\hline \multirow{2}{*}{ IBOV } & {$[11,73 ; 14,52]$} & 14,28 \\
\hline USDBRL & {$[13,02 ; 15,65]$} & 15,05 \\
\hline ABEV3 & {$[9,74 ; 12,01]$} & 9,92 \\
\hline GGBR4 & {$[6,51 ; 7,66]$} & 6,98 \\
\hline VALE3 & {$[18,56 ; 21,55]$} & 18,57 \\
\hline PETR3 & {$[22,05 ; 26,68]$} & 23,48 \\
\hline YDUQ3 & {$[20,42 ; 25,21]$} & 20,46 \\
\hline
\end{tabular}

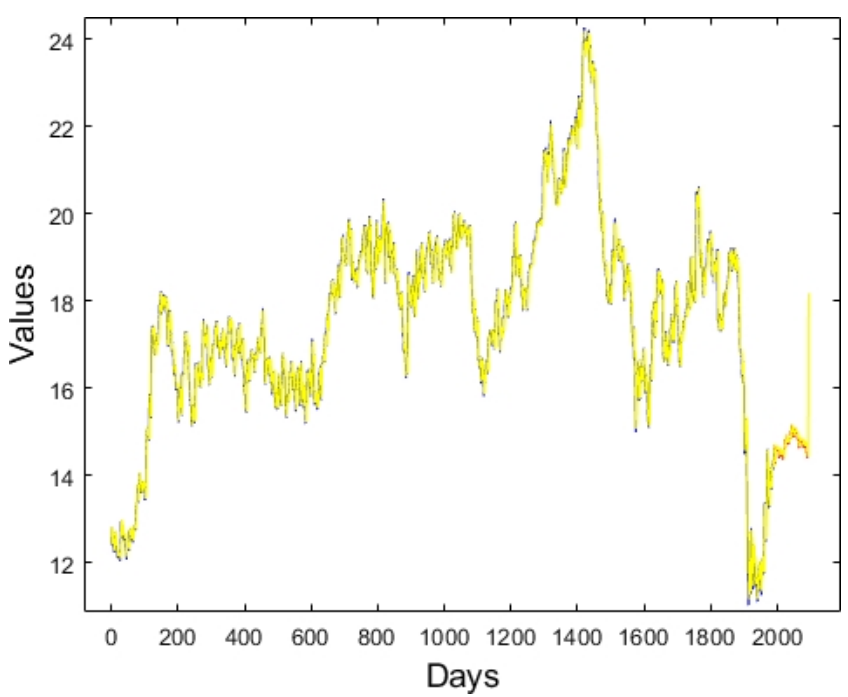

Fig. 1. Ambev historical value.

within the expected, which shows that the model is convincing for longer-term analyzes.

To exemplify the forecasting model, two historical series of share values and the relationship between the value of the Dollar in relation to the Brazilian Real on the stock exchange were taken. Figures 1. 2. and 3 shows the historical series of the Ambev's stock actions and with zoom, where it is possible to see that in the historical simulation followed by the random generation of 100 days, it visually follows the expected value in the future, however, when the dollar value is shown, shown in Figure 4 it is noticed that the algorithm cannot follow the historical value and not even the values of the 100-day simulation. In Figure 4 the yellow line represents the real historical value of the dollar, the blue line shows the model's monitoring, and finally the red line for the 100-day simulation. It can be seen in Figure 1 that the lines of historical analysis and simulation are mixed and can be perceived only with the zoom, which is shown in Figure 3 


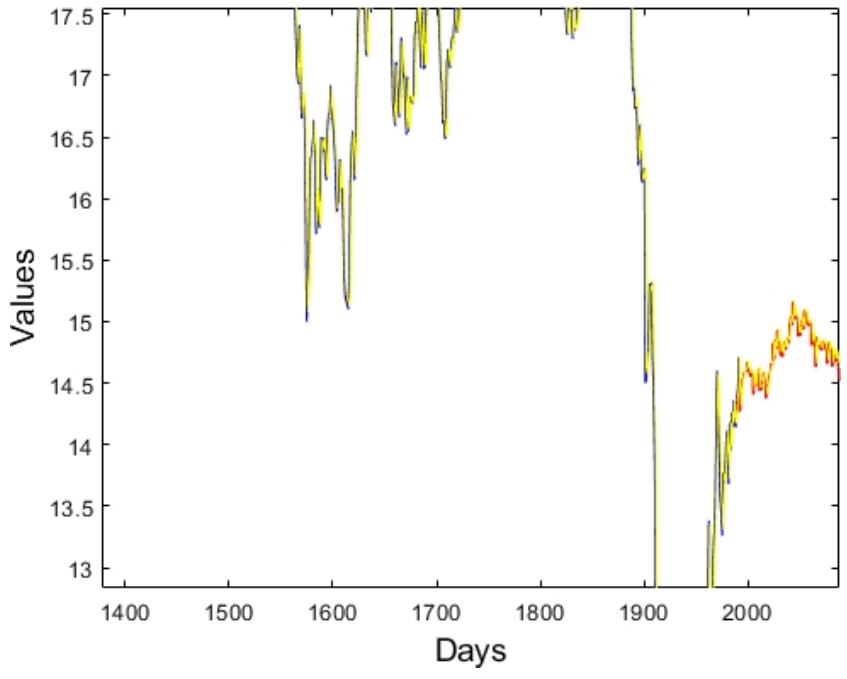

Fig. 2. Ambev historical value with zoom.

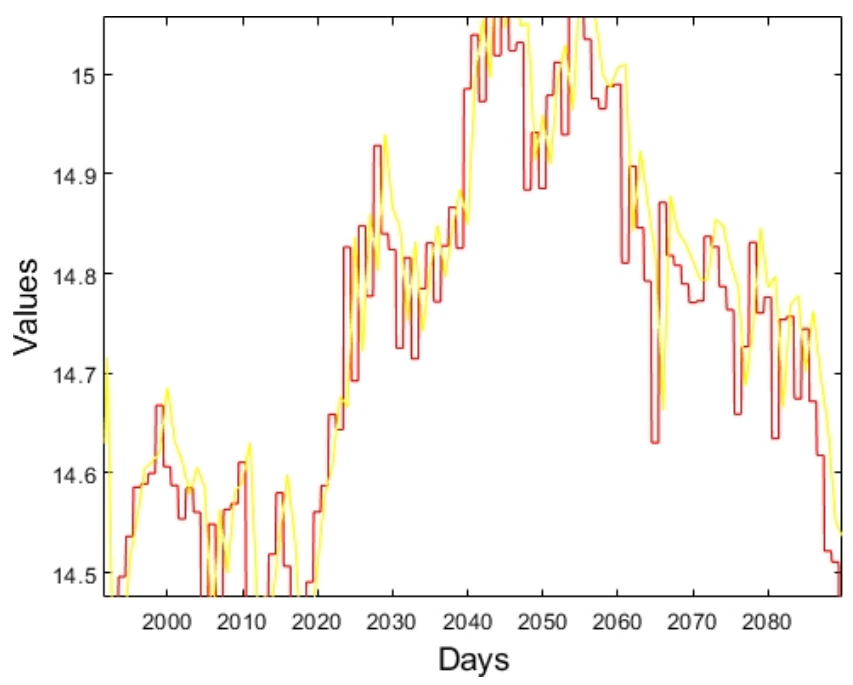

Fig. 3. Ambev historical value with zoom detail.

\subsection{Comparison of indexes in models}

After analyzing all the data, in order to obtain an estimate for values that occurred historically, considering mainly that the waiting time would be unfeasible for future analyzes, we opted to generate massive random analyzes using the Monte Carlo method associated with the Brownian Movement, considering an analysis as if the model was in use in the past, what was obtained was a very realistic approximation of the movements on the stock exchange. The figure 5 it presents what was obtained in response, where the yellow line represents the index analyzed, the blue line the Brownian motion algorithm, and the red line a mixture between the two, which uses the stock value the previous day to calculate the next step. The Figure shows that the approximation is very close to reality and that, even if it is a probabilistic analysis, it allows us to know with a high rate of accuracy whether there will be an increase or decrease in the value, even considering a decision variable on uncertainty totally unpredictable.

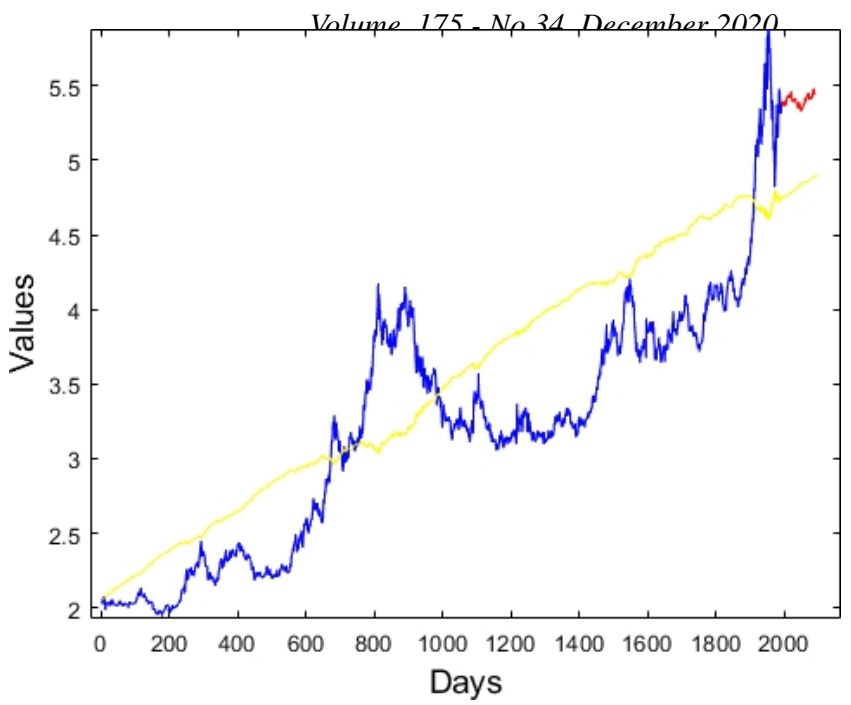

Fig. 4. Historical value Dollar.

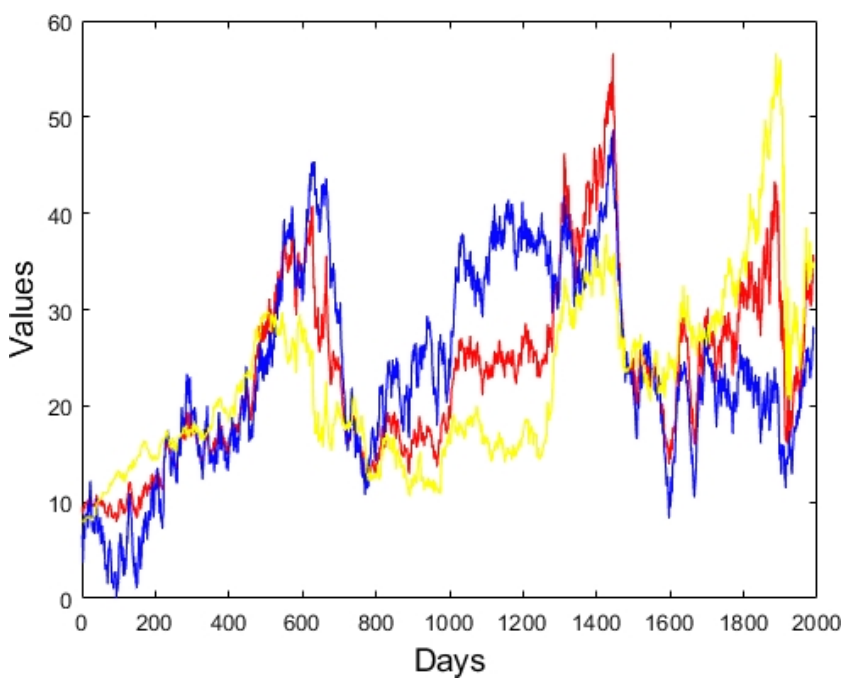

Fig. 5. Comparison of indexes in models.

\section{CONCLUSION}

The model implemented in this work serves as a source of information and a decision support system, under no circumstances should it be used as the only source of information and guarantee of results. The model serves for analysis and to generate knowledge.

This is due to the simple reason that under no circumstances is there $100 \%$ certainty in random movements, and if so, it would not be necessary to predict since there would be certain of the conclusion. As a source of consultation, the model proved to be quite stable and its reasoning should be verified over a period.

The generation of future values with the Monte Carlo Method associated with that of the Brownian Movement proved to be very efficient for simulating stock exchange values, specifically for stocks. The same did not happen with the forecast for currency values and for short-term investments, and this still needs to be adjusted. There is still a need to verify if the model will be efficient based on the future analysis of the values studied in this work and even so, it 
is still necessary to analyze all the possible impacts of events and disasters such as COVID-19 and how to proceed with the model, in this case, even so, the algorithm can serve as a great source of consultation to support the decisions of investors with little experience on the stock exchange.

Thus, the model and implementation in MatLab, in addition to serving to study the movement of the stock market, can be used in several forecasting systems, from the stock market, weather conditions, profit analysis, product launch, among others, it is enough to obtain an initial estimate based on some previously recorded knowledge.

It is important that there is knowledge recorded with historical data collection for the model to work, as the random generation in the implementation is done within a range that varies between the smallest and largest increase value between 2 consecutive days. Finally, it is expected that this model will be used as a basis for studying other random events of uncertainty.

\subsection{Future works}

As future work, we propose to study the results obtained and compare them with the real values of the actions of the future. In addition, it is proposed to apply the model in other analyzes such as climate. The third proposal for future work would be to simulate the results for other stocks and other stock exchanges, including international ones.

\section{ACKNOWLEDGMENTS}

This work was developed with financial resources from UNESA through the Research Productivity Program scholarship. I thank the Estacio de Sa University (UNESA) for funding this research and for the results achieved.

\section{REFERENCES}

[1] Mohammad Al-Shiab. The predictability of the amman stock exchange using the univariate autoregressive integrated moving average (arima) model. Journal of Economic and Administrative Sciences, 22, 122006.

[2] Francesca Biagini, Yaozhong Hu, Bernt ksendal, and Tusheng Zhang. Stochastic Calculus for Fractional Brownian Motion and Applications. Springer-Verlag London, 012008.

[3] Paolo Brandimarte. Front Matter, pages i-xvii. John Wiley \& Sons, Ltd, 2014.

[4] A. Einstein. Investigations on the Theory of the Brownian Movement. Dover Books on Physics Series. Dover Publications, 1956.

[5] Como baixar cotas de fundos de investimento da cvm para o excel.

[6] David R. Harper. How to use monte carlo simulation with gbm, Aug 2020.

[7] Ibovespa: B3.

[8] BONA L.C.E LICHT F.L., SCHULZE B. Afinidade de Tipos de Aplicacoes em Nuvens Computacionais. New Academic Editions, 062019.

[9] Modelos arima.

[10] Movimento browniano.

[11] Rizwan Mushtaq. Augmented dickey fuller test. SSRN Electronic Journal, page Augmented Dickey Fuller Test, 082011.

[12] R.Y. Rubinstein and D.P. Kroese. Simulation and the Monte Carlo Method: Third Edition. Wiley, 112016.
[13] Archived: Who timeline - covid-19. 34, December 2020 